\title{
Intrauterine ectopic pregnancy - ultrasound typing and treatment
}

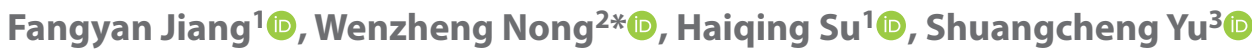 \\ ${ }^{1}$ Affiliated Ethnic Hospital of Guangxi Medical University, Departments of Ultrasound Diagnosis, China \\ ${ }^{2}$ Affiliated Ethnic Hospital of Guangxi Medical University, Department of Obstetrics and Gynecology, China \\ ${ }^{3}$ Affiliated Ethnic Hospital of Guangxi Medical University, Department of Radiology, China
}

${ }^{*}$ co-first author

\begin{abstract}
Objectives: To analyze the correlation between ultrasound typing and treatment modality of patients with an intrauterine ectopic pregnancy (cervical and cesarean scar).

Material and methods: We retrospectively enrolled 65 patients diagnosed with cesarean scar pregnancy (CSP) or cervical pregnancy (CP) between February 2014 and May 2018. The cases were divided into two types according to the ultrasound presentation with a gestational sac (GS, type I) or a heterogeneous mass (HM, type II). Type I was further divided into type la ( $<8$ weeks) and type Ib ( $\geq 8$ weeks); type II was defined as type lla (with poor or no vascularity) and type llb (with rich vascularity). Three treatment methods were applied in each group.

Results: Of included cases, there were 53 CSP and 12 CP. There was no significant difference between Type I and Type II groups in any variable. The beta human chorionic gonadotropin ( $\beta-\mathrm{hCG}$ ) level and gestational age of type llb were significantly higher compared to type lla $(p<0.05)$. There was a positive correlation between ultrasound categories and treatment methods ( $r s=0.723, p=0.000$ ). Analysis of CSP cases of initial treatment failure indicated success rate of initial dilation and curettage (D\&C) was dependent upon ultrasonic types, mean sac diameter, gestational age, hCG level, and number of cesarean sections.

Conclusions: The features of ultrasound imaging might provide an additional reference for the selection of clinical treatment methods.
\end{abstract}

Key words: cesarean scar pregnancy; cervical pregnancy; ultrasonography typing; treatment

Ginekologia Polska 2020; 91, 3: 111-116

\section{INTRODUCTION}

Cesarean scar pregnancy (CSP) and cervical pregnancy (CP) are relatively rare and unusual types of ectopic implantations in the lower segment of the uterus. They are defined as pregnancies that implant within the uterus, but outside the uterine cavity [1, 2].

Cesarean scar pregnancy occurs when a gestational sac implants at the site of a previous cesarean section. With the recent change in China's One-Child Policy to a Two-Child Policy, the incidence and detection rate of CSP are rising. Cesarean scar pregnancy incidence has increased from 1 per 2,226 to 1 per 1,800 pregnancies [3], along with an increased rate of cesarean sections and increased knowledge and awareness of diagnostic ultrasound.

In the case of $\mathrm{CP}$, the conceptus implants in the endocervical canal below the internal os. Cervical pregnancy occurs in less than $1 \%$ of all pregnancies, or 1 in 1,000 to 1 in 18,000 pregnancies [4]. The first case was reported in 1978 [5], and its risk factors have not been clearly elucidated.

The clinical symptoms of CP and CSP are similar [6]. Initially, patients may have no distinctive clinical presentation and often have mild vaginal bleeding, with or without vague abdominal pain. As pregnancy progresses, the risk of unexpected life-threatening hemorrhage or uterine rupture escalates due to the erosion of cervical blood vessels and 
invasion of the myometrium early in the first trimester [7]. On that account, timely diagnosis and individualized management are critical for reducing morbidity and mortality.

However, differentiating between CSP, CP, and threatened miscarriage via diagnostic ultrasound in a low-lying gestation sac is particularly difficult [8-10]. Not all cases of CSP and CP present with typical ultrasonic imaging [8]. Ectopic pregnancies are often accompanied by a hypoechoic mass in the lower uterine segment [11-14]. Hypoechoic masses are atypical and are sometimes misdiagnosed as anterior myometrial fibroids or trophoblastic tumors [7, 8]. At present, previous studies have mainly focused only on the gestational sac, and there is very little data on the hypoechoic mass on ultrasound findings as well as its proper management $[11,15]$.

In this report, we present an analysis of ultrasound findings and treatment strategy for patients diagnosed with CSP or CP at our institution during a 4-year period and to discuss the relationship between ultrasonography typing and treatment modality.

\section{MATERIAL AND METHODS}

This study was approved by our Institutional Review Board of Research Ethics Committee. Informed consent was obtained from all patients before they were enrolled in the study. We retrospectively included cases of CSP and CP at the Affiliated Ethnic Hospital of Guangxi Medical University from May 2014 to November 2018. Transvaginal and transabdominal ultrasound examinations were performed with an iU22 (Philips Electronics NV, Netherlands) or Hitachi EUB 6000 (Tokyo, Japan) ultrasound. The inclusion criterion for the study was the diagnosis of CP or CSP who were initially treated at our hospital. The exclusion criteria comprised the cases of $\mathrm{CP}$ or CSP who were initially taken an attempted curettage with failure in local clinics or community hospitals and subsequently transferred to our hospital, as well as abortion, heterotopic pregnancy and cornual pregnancies. The final diagnoses were established by histopathology or operative findings and were confirmed by clinical follow-up. A clinical database was compiled from information in electronic medical records including patient age, gestational age, $\beta$-hCG level at the time of primary diagnosis, initial and additional treatment.

\section{Sonographic classification of CSP and CP}

In this study, the diagnosis of CSP and CP were based on ultrasound criteria reported on the literature [1,7-9, 12]. All cases were divided into two types according to the ultrasound presentation with a gestational sac (type l) or a heterogeneous mass (type II). Then, each group was further divided into two subtypes based on gestational age or vascularity within the mass. As previously reported [16-18], the gestational age of 8 weeks is regarded as the dividing line. The

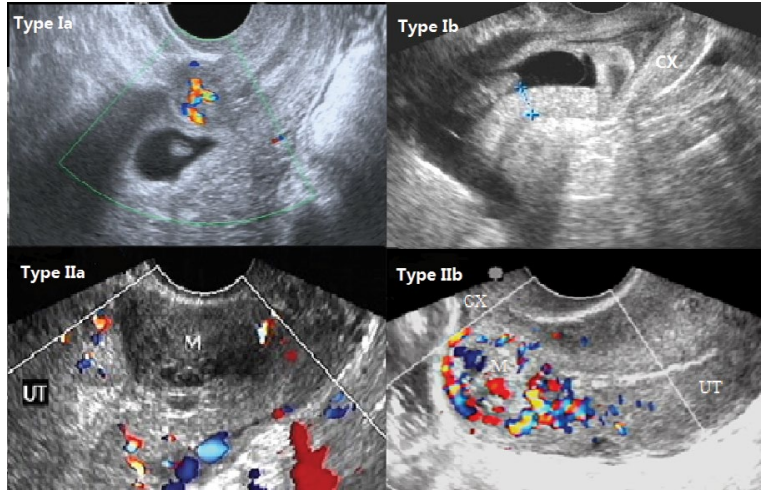

Figure 1. The description of our new ultrasound types for CSP and CP; Type la was defined as an early gestational sac ( $<8$ weeks); Type Ib represented a GS shape at $\geq 8$ weeks gestation; Type Ila indicated a heterogeneous mass without vascular pattern; Type Ilb implied a heterogeneous mass with rich trophoblastic circulation, partly generating an arteriovenous fistula

treatment of gestational sac $\geq 8$ weeks is different from the ones less than 8 weeks. Consequently, type la could be defined as a GS at $>8$ weeks and type lb represented a GS shape at $\geq 8$ weeks gestation. Similarly, type lla indicated a heterogeneous mass was highly suspected to be an ectopic pregnancy in the lower uterine segment present with poor or no vascularity on color Doppler ultrasound imaging, and type Ilb implied the heterogeneous mass in a high index of suspicion had rich vascularity by color Doppler ultrasound (Fig. 1). Two experienced radiologists (F.Y.J. and H.Q.S.) were blinded to study variables and independently categorized cases; inter-rater agreement was $100 \%$.

\section{Treatment modalities for CSP and CP}

Although many management options have been proposed, there is no consensus on the preferred mode of treatment for CSP or CP. The treatment strategies remain highly varied [19]. Three therapeutic methods were applied as initial treatment in this study. The first method, $D \& C$, was suitable for hemodynamically stable (minimal blood loss) cases of endogenous CSP and asymptomatic CP at $<8$ weeks of gestation. This method was combined with adjuvant therapy such as oral mifepristone or injection of methotrexate (MTX) and often performed under ultrasound guidance. The second method, hysteroscopic resection (HR), was often combined with additional hemostatic measures, for instance, cervical cerclage, balloon tamponade, and local injection of MTX. This treatment method was most regularly used in CP cases. Also, it was adapted for endogenous CSP patients who declined D\&C treatment. The third method, surgical resection (SR), was performed by laparoscopy, hysterotomy, or hysterectomy to cut out gestational tissues. Surgical resection was suitable for all the cases of CSP or $\mathrm{CP}$ with severe symptoms like hemodynamic instability, 
uterine rupture, or placental implantation abnormalities, as well as the cases of exogenous CSP. Surgical resection was occasionally combined with hysteroscopy and local injection of MTX. As previously mentioned, strict treatment guidelines did not exist for clinical management.

\section{Statistical analysis}

Statistical analysis was performed using SPSS version 22.0 (Chicago, IL, USA). Quantitative data was presented as mean \pm standard deviation or frequency (\%). The correlation between ultrasound types and treatment methods were calculated by Spearman correlation. One-way analysis of variance (ANOVA), Student $t$ test and Fisher's exact test were used to compare group characteristics and treatment methods. Statistical significance was defined as $p<0.05$.

\section{RESULTS}

During the 4-year period, a total of 74 patients with CSP and CP were hospitalized at our hospital. Nine patients were (eight patients with CSP, one patient with CP) excluded from this study on account of their initial treatment failed at other hospitals. Ultimately, in the ectopic pregnancies

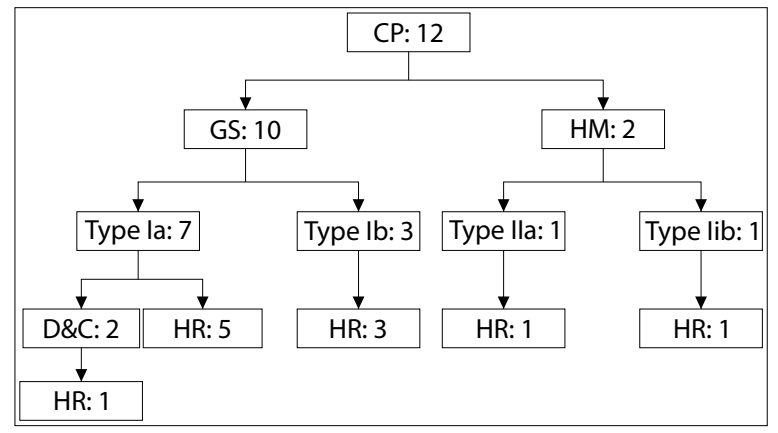

Figure 2. Ultrasound classification and treatment options for cervical pregnancy (CP); GS - gestational sac; $\mathrm{HM}$ - heterogeneous mass; D\&C — dilation and curettage; HR - hysteroscopic resection; Only one patient performed additional treatment enrolled, there were $53 \mathrm{CSP}$ and $12 \mathrm{CP}$. The mean patient age of CSP cases was $33.30 \pm 4.05$ years and the gestational age was 4-10 weeks. The mean age of CP patients was $31.00 \pm 5.75$ years and the gestational age was $4-9$ weeks.

\section{Ultrasound findings}

The ultrasound examination revealed that 45 patients presented with a gestational sac $(69.2 \%, 45 / 65)$ and 20 patients presented with a heterogeneous mass $(30.8 \%, 20 / 65)$. The numbers of patients categorized as type la, lb, Ila, and Ilb were $32,13,7$, and 13 , respectively. The detailed distribution is shown in Figure 2 and Figure 3.

No statistical difference was found between type I and type II groups with respect to patient age, $\beta$-hCG level, mean sac diameter, and gestational age at diagnosis. When ultrasonography both manifested a heterogeneous mass (type II), the average $\beta$-hCG level and gestational age of type Ilb patients were significantly higher compared to type lla cases (Tab. 1).

\section{Comparison of treatment modalities between CSP and CP}

In general, the initial treatment methods utilized in this study were $D \& C(n=31,47.7 \%)$, hysteroscopic resection

Table 1. Comparison of preoperative conditions between two ultrasound subtypes

\begin{tabular}{|l|l|l|l|}
\hline & Type Ila & Type Ilb & p \\
\hline Patient age [y] & $30.60 \pm 4.775$ & $33.89 \pm 3.333$ & 0.154 \\
\hline Gestational weeks & $5.50 \pm 1.456$ & $8.033 \pm 0.893$ & 0.002 \\
\hline $\begin{array}{l}\text { Initial hCG level } \\
\text { [milliunits/mL] }\end{array}$ & $6841.20 \pm 3259.18$ & $31577.4 \pm 21902.77$ & 0.030 \\
\hline $\begin{array}{l}\text { Gestational sac } \\
\text { size [mm] }\end{array}$ & $31.60 \pm 9.127$ & $41.16 \pm 11.89$ & 0.154 \\
\hline
\end{tabular}

Data are mean $\pm S D ; h C G$, - human chorionic gonadotropin;

Type lla - a heterogeneous mass present with poor or no vascularity; Type $\mathrm{llb}$ - a heterogeneous mass present with rich vascularity

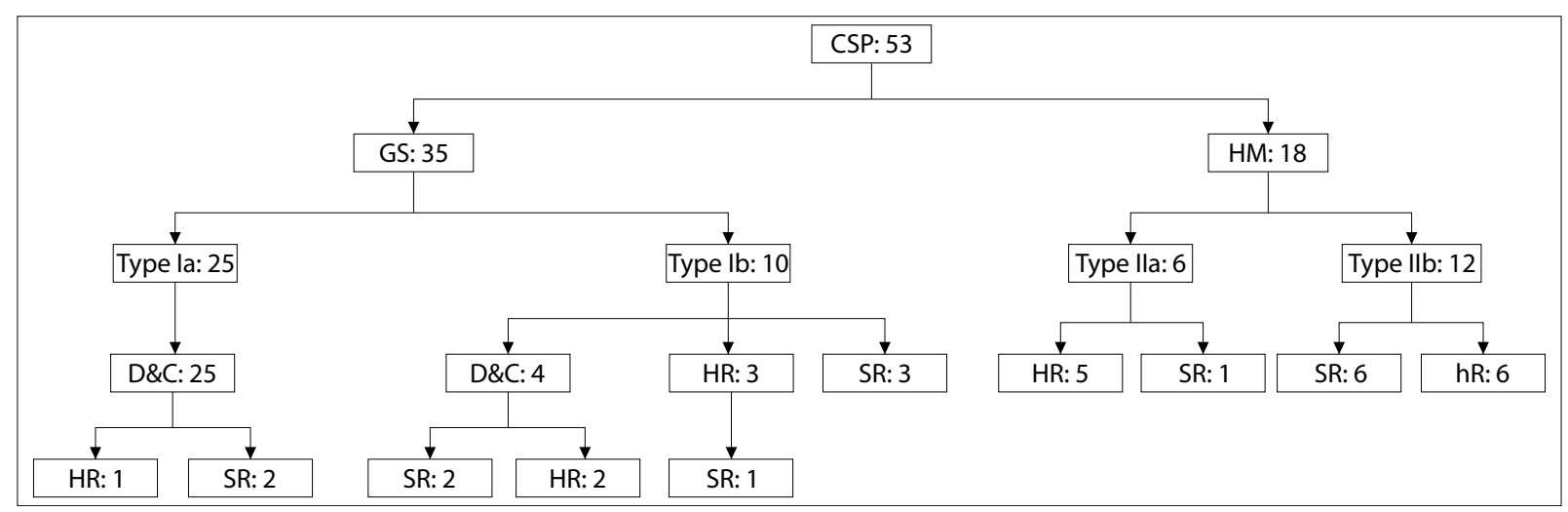

Figure 3. Ultrasound classification and treatment options for cesarean scar pregnancy (CSP); GS — gestational sac; HM — heterogeneous mass; $\mathrm{D} \& \mathrm{C}$ - dilation and curettage; HR - hysteroscopic resection; SR — surgical resection; There were 8 patients performed additional treatment 
( $n=24,36.9 \%)$, and surgical resection $(n=10,15.4 \%)$. Cervical pregnancies were mainly treated by hysteroscopic resection ( $n=10,83.3 \%), D \& C(n=2,16.7 \%)$ (Fig. 2). Cesarean scar pregnancies were primarily treated by $D \& C(n=29,54.7 \%)$, hysteroscopic resection ( $n=14,26.4 \%$ ) and surgical resection $(n=10,18.9 \%$ ) (Fig. 3). The success rate of initial treatment was 91.7\% for CP patients and $84.9 \%$ for CSP patients. The total number of cases after initial treatment failed was 9 , including $1 \mathrm{CP}$ and 8 CSP. Among of these failed cases, 8 patients were originally performed by $\mathrm{D} \& \mathrm{C}$ treatment, which were $1 \mathrm{CP}$ and 7 CSP respectively. A closer analysis of CSP failed cases revealed that successful treatment by $D \& C$ was dependent upon the ultrasound types, mean sac diameter, gestational age, $\beta$-hCG level, and number of cesarean sections (Tab. 2).

Comparing the three different treatment modalities of the ectopic pregnancy located in the lower segment of the uterus, the gestational age, mean sac diameter, and $\beta$-hCG level of patients undergoing surgical resection were significantly higher than those of patients undergoing D\&C and hysteroscopic resection (Tab. 3). By contrast, the cases of D\&C were all patients present with a gestational sac on ultrasonic imaging, along with smaller gestational age, mean sac diameter and lower $\beta$-hCG levels than the others. Spearman correlation showed that ultrasound classification was highly correlated with treatment method $(r s=0.723, p=0.000)$. The average patient age did not have significant differences among treatment modalities (Tab. 3).

\section{DISCUSSION}

It is well known that ultrasonography plays a central role in the diagnosis of ectopic pregnancies $[20,21]$. In this study, there was a notable characteristics of ultrasound findings. A proportion of enrolled cases (30.8\%) were shown heterogeneous masses upon ultrasound examination, which had less been previously reported in the literature.

Generally, the presence of an echogenic mass may indicate a different status of conception compared to a GS. It may or may not show vascularity at color Doppler US corresponding to its state, which may be related to the level of $\beta$-hCG [13]. Our data indicated the amount of vascularity (type Ila: poor vascularity, type Ilb: rich vascularity) was correlated with $\beta$-hCG level and gestational age $(p=0.030$ and $p=0.002$, respectively). Type Ilb cases presented rich vascularity surrounding a gestational trophoblastic mass. Three of these patients ( 1 CP, 2 CSP) were found arteriovenous malformations within the mass, which were easily misinterpreted as trophoblastic tumors. These observations implied that this type of mass may be an active trophoblastic tissue [13]. Conversely, our study revealed that ultrasound evidence of an avascular hypoechoic mass indicated that it was be either a degeneration of the conceptus whose serum $\beta$-hCG levels had reduced, or an subchorionic hematoma. These were consistent with surgical results.

Moreover, our data indicated a high association between ultrasound findings and treatment modalities in the first

\begin{tabular}{|l|l|l|l|}
\hline \multicolumn{5}{|l|}{ Table 2. Comparison of preoperative conditions between successful and failed treatments of CSP by dilation and curettage } \\
\hline & Success & Failure & p \\
\hline Patient age [y] & $33.0 \pm 3.57$ & $32.67 \pm 5.28$ & 0.875 \\
\hline Gestational weeks & $6.33 \pm 0.741$ & $7.767 \pm 1.041$ & 0.004 \\
\hline Initial hCG level [milliunits/mL] & $15379.17 \pm 9920.198$ & $56322.33 \pm 26271.802$ & 0.000 \\
\hline Gestational sac size [mm] & $13.633 \pm 3.671$ & $28.717 \pm 9.567$ & 0.000 \\
\hline Number of CS & $1.083 \pm 0.289$ & $1.667 \pm 0.516$ & 0.007 \\
\hline Type la & 22 & 3 & $0.001^{*}$ \\
\hline Type lb & 0 & 4 & \\
\hline
\end{tabular}

CSP — cesarean scar pregnancy; hCG — human chorionic gonadotropin; CS — cesarean sections; Data are mean \pm SD, n; * Fisher's exact test

Table 3. Comparison of preoperative conditions in three different treatment methods

\begin{tabular}{|l|l|l|l|}
\hline & Dilation and curettage & Hysteroscopic resection & Surgical resection \\
\hline Patient age [y] & $33.33 \pm 4.304(\mathrm{~A} 1)$ & $31.63 \pm 5.679(\mathrm{~A} 2)$ & $34.80 \pm 1.924(\mathrm{~A} 3)$ \\
\hline Gestational weeks & $6.69 \pm 1.149(\mathrm{~B} 1)$ & $7.01 \pm 1.695(\mathrm{~B} 2)$ & $8.53 \pm 0.612(\mathrm{~B} 3)$ \\
\hline Initial hCG level [milliunits/mL] & $28418.45 \pm 24481.593(\mathrm{C} 1)$ & $18073.79 \pm 15377.408(\mathrm{C} 2)$ & $46589.17 \pm 23771.756(\mathrm{C})$ \\
\hline Gestational sac size $[\mathrm{mm}]$ & $18.705 \pm 9.109(\mathrm{D} 1)$ & $29.484 \pm 10.175(\mathrm{D} 2)$ & $46.883 \pm 9.156(\mathrm{D} 3)$
\end{tabular}

Data are mean $\pm S D ;$ Comparisons of patient age have no statistical difference: $A 1$ vs $A 2, p=0.319 ; A 1$ vs $A 3, p=0.564 ; A 2$ vs $A 3, p=0.204 ;$ The gestational weeks indicates a significant difference among the three groups: $B 1$ vs $B 2, p=0.469 ; B 1$ vs $B 3, p=0.006 ; B 2$ vs $B 3, p=0.022$; Initial $\beta$-hCG level indicates a significant difference among the three groups: $C 1$ vs $C 2, p=0.131 ; C 1$ vs $C 3, p=0.070 ; C 2$ vs $C 3, p=0.006$; Sac size indicates a significant difference among the three groups: $D 1$ vs $D 2$, $p=0.001 ; D 1$ vs $D 3, p=0.000 ; D 2$ vs D3, $p=0.000$ 
trimester. All of the cases firstly treated by D\&C were shown a gestational sac on ultrasound findings while the patients' presence of a heterogeneous mass suggested to better initially treat with a more complex surgical procedure. This was different from the pervious studies [2, 12, 22]. In majority of reported cases, the selection of treatment modality was based on severity of symptoms, preoperative $\beta$-hCG levels, and the individual physician's preference and expertise in order to minimize possible effect on future fertile capability of the patient [18]. There was scant data on the correlation between ultrasound findings and treatment methods in the available literature.

At present, there were no consensual protocols for the treatment of CP and CSP. The management strategies of CP and CSP were somewhat different. Most patients with CP were performed by hysteroscopic resection in combination with adjuvant hemostatic techniques including balloon tamponade, cervical cerclage, and local injection of MTX. The primary management of CSP cases was D\&C and the second was hysteroscopic resection. Surgical approach was performed in only 10 patients with CSP. No patient with CP required a surgical resection.

In the present study, D\&C was the primary treatment. Although it was performed in many cases ( $n=31, C S P: 54.7 \%$, CP: $16.7 \%$ ) due to its shorter operation time, shorter hospital stay, and lower hospital cost compared to other therapeutic approaches [23], the initially unsuccessful treatment was most commonly managed with D\&C [24]. Our data showed that $88.9 \%$ (8/9) of failed cases were performed by D\&C. Particularly among them, $87.5 \%$ (7/8) of cases were CSP. This might be a factor that the success rate of initial treatment of CP was superior to CSP (91.7\% vs $84.9 \%)$. It was well-known that there were 2 types of CSP, with type 1 growing toward the uterine cavity and type 2 progressing toward the bladder and abdominal cavity [23]. D\&C treatment was ideal for type 1 CSP (endogenous CSP) to completely remove the abnormally adherent trophoblastic implantation. Unfortunately, type 2 CSP (exogenous CSP) with growth toward the bladder and abdominal cavity cannot be easily treated by D\&C [24]. It may be difficult to scrape the gestational tissue products completely because of the invasion of the myometrium and erosion of bladder early in the first trimester, which would result in the high risk of a hemorrhagic event and uterine rupture. Furthermore, we found that the patients with type la ( $<8$ weeks) were more suitable for initial D\&C treatment than type Ib ( $\geq 8$ weeks). All of type lb cases were applied additional treatment after performed D\&C. In addition, the current study revealed that larger gestational sac, higher $\beta$-hCG level, and more numbers of previous cesarean sections may increase the risk of $D \& C$ treatment failure. An earlier gestational age would likely improve $D \& C$ success rate.
This study had some limitations. Firstly, one notable limitation was retrospective nature. This influences the way in which medical data was collected and have consequently included biases as unrecognized or unmeasured factors. Secondly, 9 patients with diagnosis of CP or CSP failed by $D \& C$ treatment at other hospitals were excluded from our analysis as an attempted curettage often alters the typical ultrasound findings, which may have an effected our results. Thirdly, this study mainly summarizes the ultimate therapeutic outcomes of these patients, without detailed comparison of some relevant clinical data, such as operation time, hospital stay, and hospital cost, and hospitalization cost. Finally, this was a small-sized, single center study.

In summary, the features of ultrasound imaging may provide an additional reference for the selection of clinical treatment methods. In a high degree of suspicion of intrauterine ectopic pregnancy in the lower segment of the uterus, the patients with a gestational sac less than 8 weeks on ultrasound imaging could be initially treated by D\&C treatment while women with a heterogeneous mass in a high degree of suspicion may be better treated with a more complex surgical procedure. Future prospective and large-scale studies are warranted to validate this finding.

\section{Acknowledgements}

This work was supported by the Department of Obstetrics and Gynecology in the Affiliated Ethnic Hospital of Guangxi Medical University.

\section{REFERENCES}

1. Chukus A, Tirada N, Restrepo R, et al. Uncommon Implantation Sites of Ectopic Pregnancy: Thinking beyond the Complex Adnexal Mass. Radiographics. 2015; 35(3): 946-959, doi: 10.1148/rg.2015140202, indexed in Pubmed: 25860721

2. Jachymski T, Moczulska H, Guzowski G, et al. Conservative treatment of abnormally located intrauterine pregnancies (cervical and cesarean scar pregnancies): a multicenter analysis (Polish series). J Matern Fetal Neonatal Med. 2018; 33(6): 993-998, doi: 10.1080/14767058.2018.1514009.

3. Osborn DA, Williams TR, Craig BM. Cesarean scar pregnancy: sonographic and magnetic resonance imaging findings, complications, and treatment. J Ultrasound Med. 2012; 31(9): 1449-1456, doi: 10.7863/jum.2012.31.9.1449, indexed in Pubmed: 22922626.

4. Shavell VI, Abdallah ME, Zakaria MA, et al. Misdiagnosis of cervical ectopic pregnancy. Arch Gynecol Obstet. 2012; 285(2): 423-426, doi: 10.1007/s00404-011-1980-0, indexed in Pubmed: 21748311.

5. Raskin MM. Diagnosis of cervical pregnancy by ultrasound: a case report. Am J Obstet Gynecol. 1978; 130(2): 234-235, doi: 10.1016/00029378(78)90377-0, indexed in Pubmed: 619667.

6. Tsai SW, Huang KH, Ou YC, et al. Low-lying-implantation ectopic pregnancy: a cluster of cesarean scar, cervico-isthmus, and cervical ectopic pregnancies in the first trimester. Taiwan J Obstet Gynecol. 2013; 52(4): 505-511, doi: 10.1016/j.tjog.2013.10.009, indexed in Pubmed: 24411034.

7. Ghaneie A, Grajo JR, Derr C, et al. Unusual ectopic pregnancies: sonographic findings and implications for management. J Ultrasound Med. 2015; 34(6): 951-962, doi: 10.7863/ultra.34.6.951, indexed in Pubmed: 26014313.

8. Ravi Selvaraj L, Rose N, Ramachandran M. Pitfalls in Ultrasound Diagnosis of Cesarean Scar Pregnancy. J Obstet Gynaecol India. 2018; 68(3): 164172, doi: 10.1007/s13224-016-0956-1, indexed in Pubmed: 29895994.

9. Liu $\mathrm{D}$, Yang $\mathrm{M}, \mathrm{Wu} \mathrm{Q}$. Application of ultrasonography in the diagnosis and treatment of cesarean scar pregnancy. Clin Chim Acta. 2018; 486: 291-297, doi: 10.1016/j.cca.2018.08.012, indexed in Pubmed: 30102898. 
10. Osborn DA, Williams TR, Craig BM. Cesarean scar pregnancy: sonographic and magnetic resonance imaging findings, complications, and treatment. J Ultrasound Med. 2012; 31(9): 1449-1456, doi: 10.7863/jum.2012.31.9.1449, indexed in Pubmed: 22922626.

11. Takahashi $H$, Usui $R$, Suzuki $H$, et al. Uterine-fundal hypoechoic mass: a possible ultrasound sign for cesarean scar pregnancy. Clin Exp Obstet Gynecol. 2017; 44(1): 88-92, indexed in Pubmed: 29714873.

12. Samal SK, Rathod S. Cervical ectopic pregnancy. J Nat Sci Biol Med. 2015; 6(1): 257-260, doi: 10.4103/0976-9668.149221, indexed in Pubmed: 25810679.

13. Api $\mathrm{O}$, Unal $\mathrm{O}$, Api $\mathrm{M}$, et al. Ultrasonographic appearance of cervical pregnancy following successful treatment with methotrexate. Ultrasound Obstet Gynecol. 2006; 28(6): 845-847, doi: 10.1002/uog.3846, indexed in Pubmed: 17063447.

14. OuYang $Z$, Yin $Q, X u Y$, et al. Heterotopic cesarean scar pregnancy: diagnosis, treatment, and prognosis. J Ultrasound Med. 2014; 33(9): 1533-1537, doi: 10.7863/ultra.33.9.1533, indexed in Pubmed: 25154932.

15. Sun $\mathrm{QL}, \mathrm{Wu} X \mathrm{H}$, Luo Li, et al. Characteristics of women with mixed mass formation after evacuation following uterine artery chemoembolization for cesarean scar pregnancy. Arch Gynecol Obstet. 2018; 297(4): 10591066, doi: 10.1007/s00404-018-4716-6, indexed in Pubmed: 29450694.

16. Birch Petersen K, Hoffmann E, Rifbjerg Larsen C, et al. Cesarean scar pregnancy: a systematic review of treatment studies. Fertil Steril. 2016; 105(4): 958-967, doi: 10.1016/j.fertnstert.2015.12.130, indexed in Pubmed: 26794422.

17. Gao L, Huang Z, Gao J, et al. Uterine artery embolization followed by dilation and curettage within 24 hours compared with systemic metho- trexate for cesarean scar pregnancy. Int J Gynaecol Obstet. 2014; 127(2): 147-151, doi: 10.1016/j.ijgo.2014.05.005, indexed in Pubmed: 25212971.

18. Le A, Li M, XuY, et al. Different Surgical Approaches to 313 Cesarean Scar Pregnancies. J Minim Invasive Gynecol. 2019; 26(1): 148-152, doi: 10.1016/j.jmig.2018.03.035, indexed in Pubmed: 29758363.

19. Maheux-Lacroix S, Li F, Bujold E, et al. Cesarean Scar Pregnancies: A Systematic Review of Treatment Options. J Minim Invasive Gynecol. 2017; 24(6): 915-925, doi: 10.1016/j.jmig.2017.05.019, indexed in Pubmed: 28599886.

20. Monteagudo A, Romero JA, Timor-Tritsch IE. Pregnancy in an Abnormal Location. Clin Obstet Gynecol. 2017; 60(3): 586-595, doi: 10.1097/GRF.0000000000000306, indexed in Pubmed: 28742592.

21. Rotas MA, Haberman S, Levgur M. Cesarean scar ectopic pregnancies: etiology, diagnosis, and management. Obstet Gynecol. 2006; 107(6): 1373-1381, doi: 10.1097/01.AOG.0000218690.24494.ce, indexed in Pubmed: 16738166.

22. Moschos E, Wells CE, Twickler DM. Biometric sonographic findings of abnormally adherent trophoblastic implantations on cesarean delivery scars. J Ultrasound Med. 2014; 33(3): 475-481, doi: 10.7863/ultra.33.3.475, indexed in Pubmed: 24567459.

23. Zhang $\mathrm{H}$, Huang J, Wu $\mathrm{X}$, et al. Clinical classification and treatment of cesarean scar pregnancy. J Obstet Gynaecol Res. 2017; 43(4): 653-661, doi: 10.1111/jog.13267, indexed in Pubmed: 28150370.

24. Wu XQ, Zhang HW, Fang XL, et al. Factors associated with successful transabdominal sonography-guided dilation and curettage for early cesarean scar pregnancy. Int J Gynaecol Obstet. 2015; 131(3): 281-284, doi: 10.1016/j.ijgo.2015.06.029, indexed in Pubmed: 26372348. 